\title{
Chest-wall Surface Dose During Post-mastectomy Radiation Therapy, with and without Nonmagnetic Bolus: A Phantom Study
}

\author{
Cheon Woong Choi ${ }^{1}$, Joo Wan Hong ${ }^{2,4}$, Cheol Soo Park ${ }^{3}$, and Jae Ouk Ahn ${ }^{4 *}$ \\ ${ }^{1}$ Department of Respiratory and Critical Care Medicine, Kyung Hee University Hospital at Gangdong, Seoul 05278, Korea \\ ${ }^{2}$ Department of Radiation Oncology, Seoul National University Bundang Hospital, Seongnam 13620, Korea \\ ${ }^{3}$ Department of Radiological Science, Hallym Polytechnic University, Chuncheon 24210, Korea \\ ${ }^{4}$ Department of Medical IT Engineering, Soonchunhyang University, Asan 31538, Korea
}

(Received 28 April 2016, Received in final form 7 June 2016, Accepted 7 June 2016)

\begin{abstract}
For mastectomy patients, sufficient doses of radiation should be delivered to the surface of the chest wall to prevent recurrence. A bolus is used to increase the surface dose on the chest wall, whereby the surface dose is confirmed with the use of a virtual bolus during the computerized treatment-planning process. The purpose of this study is an examination of the difference between the dose of the computerized treatment plan and the dose that is measured on the bolus. Part of the left breast of an Anderson Rando phantom was removed, followed by the attainment of computed tomography (CT) images that were used as the basis for computerized treatment plans that were established with no bolus, a $3 \mathrm{~mm}$-thick bolus, a $5 \mathrm{~mm}$-thick bolus, and a $10 \mathrm{~mm}$-thick bolus. For the computerized treatment plan, a prescribed dose regimen was dispensed daily and planning target volume (PTV) coverage was applied according to the RTOG 1304 guidelines. Using each of the established computerized treatment plans, chest-wall doses of 5 points were measured; this chest-wall dose was used as the standard for the analysis of this study, while the level of significance was set at $P<0.05$. The measurement of the chest-wall dose with no bolus is $1.6 \%$ to $10.3 \%$ higher, and the differences of the minimum average and the maximum average of the five measurement points are -13.8 and -1.9 , respectively $(P<0.05)$; however, when the bolus was used, the dosage was measured as $3.7 \%$ to $9.2 \%$ lower, and the differences of the minimum average and the maximum average are 7.4 and $9.0,-1.2$ and 17.4 , and 8.1 and 19.8 for $3 \mathrm{~mm}, 5 \mathrm{~mm}$, and $10 \mathrm{~mm}$, respectively $(P<0.05)$. As the thickness of the bolus is increased, the differences of the average surface dose are further increased. There are a variety of factors that affect the surface dose on the chest wall during post-mastectomy radiation therapy, for which verification is required; in particular, a consideration of the appropriate thickness and the number of uses when a bolus is used, and which has the greatest effect on the surface dose on the chest wall, is considered necessary.
\end{abstract}

Keywords : nonmagnetic bolus, post-mastectomy, bolus, chest wall, surface dose

\section{Introduction}

In addition to surgery and anti-cancer chemotherapy, radiation therapy is a very important breast-cancer treatment and is performed in a variety of ways to prevent a local recurrence of the cancer; therefore, radiation therapy has recently been introduced as a basic therapy for breast cancer along with surgery [1-5]. The establishment of a radiation-therapy radiation-treatment plan for breast-cancer patients who have undergone a mastectomy is complicated because the breast tissues have been completely removed;

(C)The Korean Magnetics Society. All rights reserved.

*Corresponding author: Tel: +82-41-530-1365

Fax: +82-41-530-3084, e-mail: jahn1365@gmail.com furthermore, a corresponding insufficient surface dose is likely to be due to a build-up phenomenon that is caused by a radiation-treatment plan that comprises high-energy photons on the chest wall and has been established for post-mastectomy patients with no actual breast tissue left [6]. Under these circumstances, the most common method for increasing the chest-wall surface dose while also suppressing the likelihood of a local recurrence is the attachment of a bolus, a tissue-equivalent material, to the chest wall within the range of the radiation treatment. Boluses are used for a variety of purposes such as the increasing of the surface dose during radiation therapy and the compensation of the tissue-loss area. The main purpose of bolus usage, however, is the increasing of the surface dose for a tumor that is located on the surface of 
the head, neck, and skin to reduce the depth dose during electron-beam radiation treatment; moreover, a bolus is used in breast-cancer radiation therapy for patients who have undergone a mastectomy during photon treatment, whereby it is used to increase the surface dose and reduce the depth dose, akin to the functioning of a bolus during electron-beam treatment [7-9]. A bolus is therefore directly attached to the chest wall to increase the surface dose that is applied for the prevention of a local recurrence during radiation therapy, and it can be either attached to the entire chest wall or only to the vicinity of the surgical scars [10]. Although boluses are used to prevent a local recurrence through the increase of the surface dose [11, 12], such an increase is likely to result in a variety of side effects including the typical radiation-mediated dermatitis [1, 13-15]. During the simulated radiation-treatment planning for mastectomy patients, CT (computed tomography) images are not acquired in the presence of the bolus that is attached to the chest wall of the patient. Instead, a virtual bolus is formed during the computerized radiation treatment with a plan that the radiation-dose changes will be predicted accordingly; however, it is difficult to calculate both the high-energy photon and the measurement of the radiation dose on the thin area and surface [16]. The verification of the surface dose, which is measured using the same treatment plan that applies to the established chest-wall surface dose, through the formation of a virtual bolus is therefore required during the planning of the computerized radiation treatment. In consideration of the previously mentioned information, the aim of this study is the validation of both the surface dose when a virtual bolus is used and the surface dose on the chest wall, the latter of which is a build-up area when an actual bolus is used.

\section{Materials and Methods}

\subsection{Acquisition of CT image}

To obtain the CT images for the computerized treatment plan, an Alderson-Rando phantom (RSD Radiology Support Devices, California, U.S.A.) was used. The AldersonRando phantom contains the basic human anatomical structures such as skeleton structure, lung, and heart, and for the phantom that was used in our experiment, only the upper body was used after the phantom of the left breast tissues was removed to mimic the conditions of a mastectomy. Philips's Brilliance Big Bore CT scanner (Philips medical system, Nederland BV) was used and the CTscan conditions were set to $120 \mathrm{kV}, 50 \mathrm{~mA}, 250 \mathrm{mAs}$, a 5 $\mathrm{mm}$ thickness, and a $600 \mathrm{~mm}$ FOV. The CT-scan ranges encompass a sufficient inframammary skin fold from the

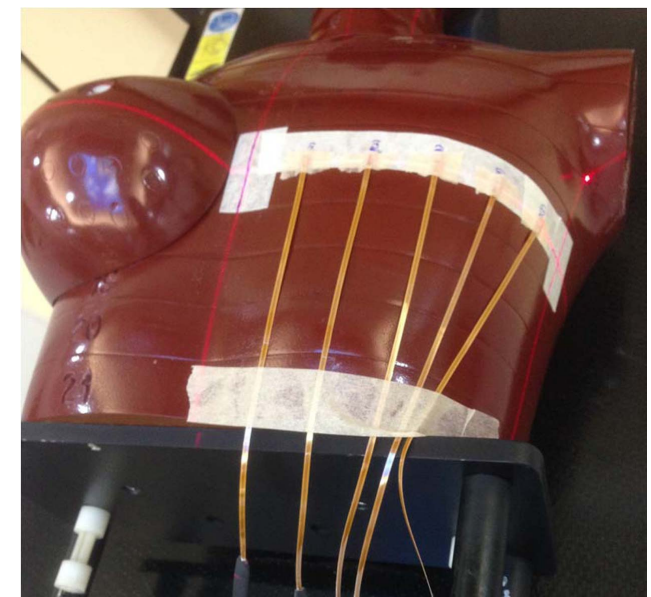

Fig. 1. (Color online) Illustration of the Alderson-Rando phantom with the MOSFET-measurement positions marked.

mandible in accordance with the RTOG 1304 guidelines that includes both lungs. After a total of five TN-502RD MOSFETs (metal-oxide-semiconductor field-effector transistors) were attached to $3 \mathrm{~cm}$-interval positions that start $3 \mathrm{~cm}$ away from the phantom median plane toward the direction of the median axillary line, the scanning was performed (Fig. 1).

\subsection{Radiation Treatment Plan}

The Pinnacle (version 9.3, Philips Medical System,

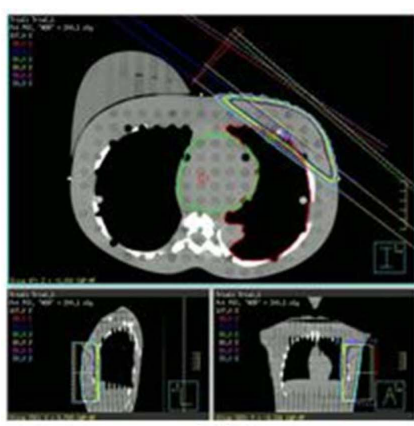

(A)

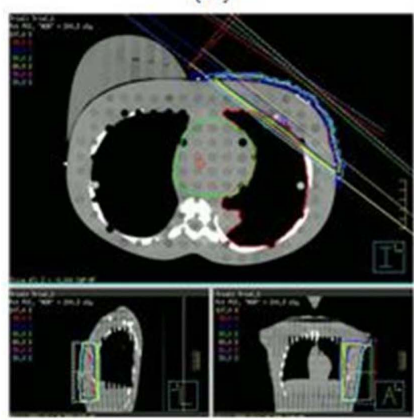

(C)

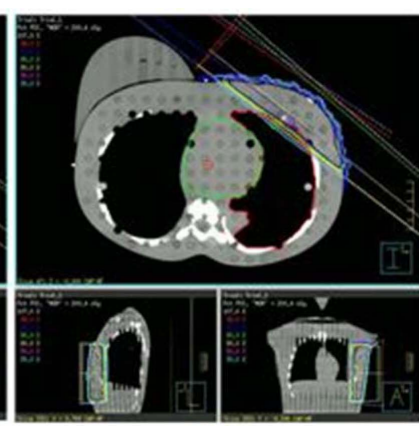

(B)

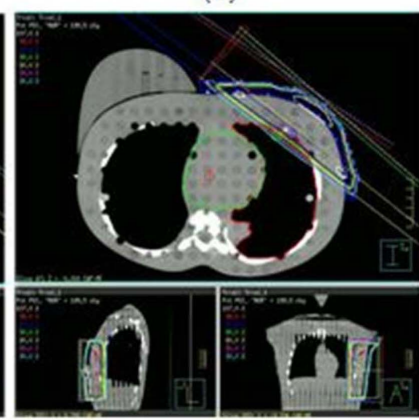

(D)
Fig. 2. (Color online) Dose distribution displayed for nonbolus and each bolus. 
U.S.A.) treatment-planning system (TPS) was used for the computerized radiation-treatment plan that comprises a daily dose of 200 cGy regimen that was prescribed using a $6 \mathrm{MV}$ Photon. The treatment plan includes a Planning Target Volume (PTV) of at least $95 \%$ on the left chest wall. The SI (superioinferior, Y field) radiation field was set to $18 \mathrm{~cm}$, and the lateral (X filed) radiation field was set to $6 \mathrm{~cm}$ with a margin of $2 \mathrm{~cm}$ to the anterior direction so that it contains enough chest wall. The gantry angles of $308^{\circ}, 128^{\circ}$, and $20^{\circ}$ of the Enhanced Dynamic Wedge (EDW) were set so that all of the chest-wall tissues are included, and the lung and heart were given the minimum radiation doses. The Arm 2 radiation-therapy group2 was set as the standard for the radiation-treatment plan in accordance with the NSABP B-51/RTOG 1304 guidelines [17]. The virtual bolus of the TPS was applied so that the non-bolus and the $3 \mathrm{~mm}, 5 \mathrm{~mm}$, and $10 \mathrm{~mm}$ thicknesses were all fully included in the radiation field of the chest wall, for which the Adaptive Convolve algorithm was used to establish four radiation-treatment plans (Fig. 2 ). The bolus density of the TPS was set to $1 \mathrm{~g} / \mathrm{cm}^{3}$.

\subsection{Measurement of radiation dose}

A linear accelerator (21EX-S, VARIAN, California, U.S.A.) was used to measure the surface dose on the chest wall; akin to the CT scan that was performed on the Alderson-Rando phantom, five TN-502RD MOSFET detectors were attached to the same positions on the chest wall. The surface dose on the chest wall was measured either without the use of a bolus or with the attached boluses of different thickness (Fig. 3). The MOSFET detectors were used for the measurement of the chest-wall surface dose, and the calibration of the standard bias mode was performed beforehand. For the calibration, the $200 \mathrm{cGy}$ of the $6 \mathrm{MV}$ photon with a depth of $1.5 \mathrm{~cm}$ was irradiated using the MOSFET-specific phantom, and the solid water phantom allowed for the calculation of the calibration factor (CF) for each detector whereby the

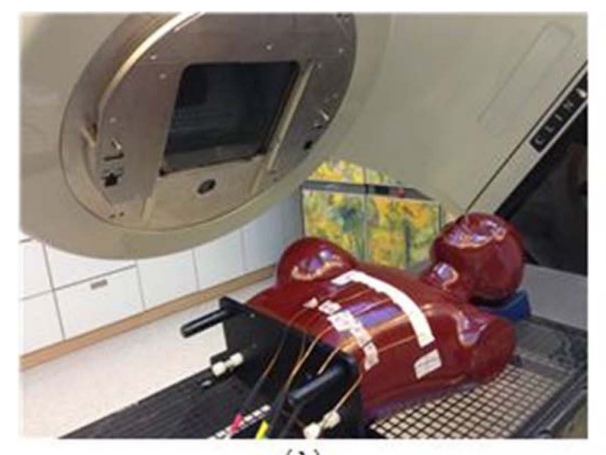

(A) following CF-calculation formula is used:

$$
\mathrm{CF}=\frac{\text { MOSFET } \mathrm{mV} \text { reading }(\mathrm{mV})}{\text { Known radiation value }(\mathrm{cGy})}
$$

\subsection{Metal-Oxide-Semiconductor Field-Effector Tran- sistor (MOSFET)}

The MOSFET that was used for this experiment is a Pchannel semiconductor-measurement instrument that is of a size that is less than $4 \mathrm{~mm}$; with excellent linearity and reproducibility, the semiconductor directly measures a radiation dose by using a very thin reaction area of less than $2 \mu \mathrm{m}$ (A picture of a MOSFET will be prepared and inserted). There is no energy dependency here, and the dependency of the radiation-incidence angle is $\pm 2 \%$ at an energy range of $1 \mathrm{MeV}$ to $20 \mathrm{MeV}$ with a $360^{\circ}$ rotation. Also, a temperature dependency of $0.5 \%$ exists at $20^{\circ}$ to $40^{\circ}$ and unlike the TLD, the influence of the temperature change is low, which is useful for the measurement of the accumulated radiation dose [18, 19]. Threshold voltage $\left(\mathrm{V}_{\mathrm{T}}\right)$ is required for a current to flow into the MOSFET and the general threshold voltage is the sum of the flat band voltage (VFB) and the ideal threshold voltage ( $\mathrm{V}_{\mathrm{T}}^{\text {ideal }}$ ), as follows:

$$
\begin{aligned}
& \mathrm{V}_{\mathrm{T}}=\mathrm{V}_{\mathrm{FB}}+\mathrm{V}^{\text {ideal }} \\
& \text { Here, } \mathrm{V}_{\mathrm{FB}}=\mathrm{e}_{\mathrm{ms}}-\frac{Q i}{C i} \\
& \mathrm{~V}_{\mathrm{T}}^{\text {ideal }}=-\frac{Q s}{C i}+2{ }_{\mathrm{F}}
\end{aligned}
$$

where $\Phi_{\mathrm{ms}}$ is the work-function difference between a metal and a semiconductor; $Q_{i}$ is the amount of charge for the oxidized insulation layer; $\mathrm{C}_{\mathrm{i}}$ is the capacitance of the oxide-insulating layer; Qs is the sum of the charges of both the depletion layer and the inversion layer; and ${ }_{F}$ is the Fermi potential.

In such a MOSFET, the threshold voltage is changed before and after irradiation due to the positive hole during

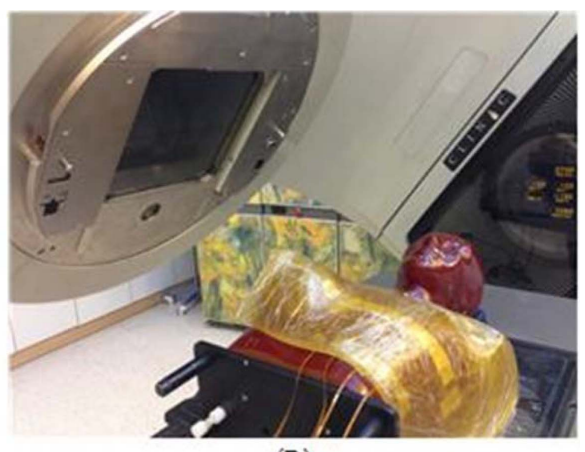

(B)

Fig. 3. (Color online) Measurement of chest-wall surface dose. 
irradiation, and the changed threshold voltage is proportional to the irradiated dose, from which the irradiation dose can be measured [20]. A comparative analysis of the surface dose on the chest wall, depending on whether or not a bolus is applied and the thickness of the bolus, was performed and the computerized treatment plan was used as the basis. The SPSS 20.0 (SPSS for Windows, ver 20.0. SPSS Inc., Illinois, U.S.A. Chicago) statistical program was used for the data analysis of the measured radiation dose, the ANOVA was used for the analysis, and Tukey's method was used for the post-analysis. The significance level was considered at $p<0.05$.

\section{Results}

The surface dose on the chest wall was measured using a MOSFET and the result confirmed the different radiation doses of the computerized treatment plan and the measured dose $(p<0.05)$, as shown in Fig. 4. Without using a bolus, the surface-dose measurement on the chest wall is $1.6 \%$ to $10.3 \%$ higher than that of the computerized treatment plan, whereby the differences of the minimum and maximum averages of the five measurement points are -13.8 and $-1.9(p<0.05)$, respectively. When a bolus was used, the increase of the surface dose on the chest wall was higher than the no-bolus results in all of the experiments; however, the surface-dose measurements are $3.7 \%$ to $9.2 \%$ lower than the computerized treatment plan at all of the measurement points, with the exception of the No. 1 detector of the $5 \mathrm{~mm}$-thick bolus. The differ-

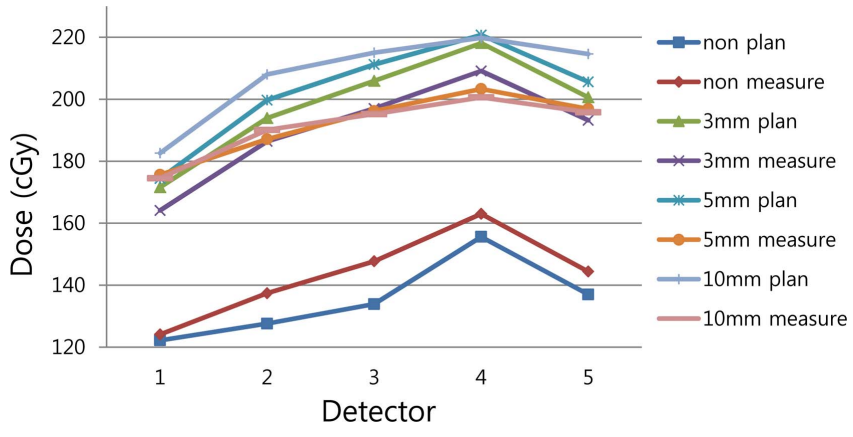

Fig. 4. (Color online) Dose comparisons of treatment planning and measurements, with and without bolus.

ences of the minimum and maximum averages of the five measurement points are 7.4 and $9.0,-1.2$ and 174 , and 8.1 and $19.8(P<0.05)$ for the $3 \mathrm{~mm}-, 5 \mathrm{~mm}-$, and 10 mm-thick boluses, respectively (Table 1 ). In addition, as the thickness of the bolus increased, the differences of the average surface doses that were measured at each measurement point were further increased (Table 2).

\section{Discussion}

After the mastectomy, the surface dose on the chest wall was measured and analyzed depending on whether a bolus was used or not and the thickness that was used in the radiation therapy. In a comparison with the computer treatment plan, it was confirmed that the measured surface dose is higher when a bolus was not used; conversely, the measured surface dose is lower than that of the computer

Table 1. Comparisons of treatment planning and average chest-wall surface doses for each MOSFET detector $(n=30)$.

\begin{tabular}{|c|c|c|c|c|c|c|c|c|c|c|}
\hline & \multicolumn{2}{|c|}{1} & \multicolumn{2}{|c|}{2} & \multicolumn{2}{|c|}{3} & \multicolumn{2}{|c|}{4} & \multicolumn{2}{|c|}{5} \\
\hline & Plan & Meas. & Plan & Meas. & Plan & Meas. & Plan & Meas. & Plan & Meas \\
\hline Non & 122.2 & 124.1 & 127.6 & 137.4 & 133.9 & 147.7 & 155.6 & 163.0 & 137.0 & 144.4 \\
\hline $3 \mathrm{~mm}$ & 171.6 & 164.1 & 193.9 & 186.4 & 205.9 & 197.0 & 218.1 & 209.1 & 200.6 & 193.2 \\
\hline $5 \mathrm{~mm}$ & 174.4 & 175.6 & 199.7 & 187.1 & 211.2 & 196.2 & 220.7 & 203.3 & 205.6 & 196.9 \\
\hline $10 \mathrm{~mm}$ & 182.6 & 174.5 & 208.0 & 190.1 & 215.0 & 195.2 & 219.8 & 200.6 & 214.6 & 195.8 \\
\hline
\end{tabular}

$(p<0.05)$

Abbreviations: Meas. $=$ measured

Table 2. Comparisons of treatment planning and measurements of mean difference and standard deviation for each MOSFET detector $(n=30)$.

\begin{tabular}{|c|c|c|c|c|c|c|c|c|c|c|}
\hline & \multicolumn{2}{|c|}{1} & \multicolumn{2}{|c|}{2} & \multicolumn{2}{|c|}{3} & \multicolumn{2}{|c|}{4} & \multicolumn{2}{|c|}{5} \\
\hline & MD & SD & MD & SD & MD & SD & MD & SD & MD & SD \\
\hline Non & -1.9 & 1.605 & -9.8 & 1.037 & -13.8 & 3.612 & -7.4 & 0.910 & -7.4 & 1.522 \\
\hline $3 \mathrm{~mm}$ & 7.5 & 0.845 & 7.5 & 2.811 & 8.9 & 0.643 & 9.0 & 1.062 & 7.4 & 0.610 \\
\hline $5 \mathrm{~mm}$ & -1.2 & 0.498 & 12.6 & 1.470 & 15.0 & 1.270 & 17.4 & 0.466 & 8.7 & 2.249 \\
\hline $10 \mathrm{~mm}$ & 8.1 & 1.456 & 17.9 & 1.062 & 19.8 & 0.887 & 19.2 & 1.037 & 18.8 & 1.808 \\
\hline
\end{tabular}

$(p<0.05)$

Abbreviations: $\mathrm{MD}=$ mean difference $\mathrm{SD}=$ standard deviation 
treatment plan when a bolus was used. These results indicate that, although a lower surface dose was expected because the relatively thin chest wall becomes part of the build-up area of the $6 \mathrm{MV} \mathrm{X}$-ray after the removal of the breast tissues, there are a variety of factors that affect the surface dose [21]. In addition, the difference between the surface doses on the chest wall of the computerized treatment plan and those of the actual measurement was reported in the previous experiment, and there is a MOSFETderived $\pm 6 \%$ surface-dose-measurement difference that confirms a similarity to this study [22,23]. It was shown that as the curvature of the region that the MOSFETmeasuring position is located in increases, the radiation dose increases in the same cross-section, and this tendency is attributed to the angle dependency of the MOSFET itself according to the attachment position [18, 24]. In addition, we confirmed that, although the absorbed dose in the build-up area is very important for tangential irradiation, one of the radiation-therapy techniques for breast cancer, there is a radiation-dose difference that depends on the radiation-dose-calculation algorithm of the instrument that is used for the computerized treatment plan $[25,26]$; therefore, an additional verification of the treatment plan that uses algorithms that are different from the adaptive convolution algorithm that was used in this experiment is needed. Regarding post-mastectomy radiation therapy for the chest wall, the recommendation stipulates the delivery of at least $80 \%$ of the prescribed dose for the surface dose on the chest wall [27]; however, a lesser dose than what is recommended was delivered to most of the points when a bolus was not used.

\section{Conclusion}

The use of a bolus of a proper thickness and with an adequate number of uses is therefore needed for postmastectomy radiation therapy, because a sufficient radiation dose should be delivered to the surface of the chest wall to address the corresponding high recurrence rate.

\section{Acknowledgement}

Cheon Woong Choi and Joo-Wan Hong equally contributed to this work. This work was supported in part bythe Soonchunhyang University Research Fund.

\section{References}

[1] A. Recht, S. B. Edge, L. J. Solin, D. S. Robinson, A. Estabrook, and R. E. Fine, J. Clin. Oncol. 19, 1539 (2001).

[2] M. Overgaard, H. M. Nielsen, and J. Overgaard, Radiother Oncol. 82, 247 (2007).
[3] J. R. Harris, P. Halpin-Murphy, M. McNeese, N. P. Mendenhall, M. Morrow, and N. J. Robert, Int. J. Radiat. Oncol. Biol. Phys. 44, 989 (1999).

[4] J. Kurtz, Eur. J. Cancer. 38, 1961 (2002).

[5] P. T. Truong, I. A. Olivotto, T. J. Whelan, and M. Levine, CMAJ. 170, 1263 (2004).

[6] M. Fischbach, R. Halg, M. Hartmann, J. Besserer, G. Gruber, and U. Schneider, Radiat Oncol. 8, 270 (2013).

[7] R. J. Kudchadker, J. A. Antolak, W. H. Morrison, P. F. Wong, and K. R. Hogstrom, J. Appl. Clin. Med. Phys./ Amer. Coll. Medi. Phys. 4, 321 (2003).

[8] S. H. Hsu, P. L. Roberson, Y. Chen, R. B. Marsh, L. J. Pierce, and J. M. Moran, Phys. Med. Biol. 53, 2593 (2008).

[9] B. Demir, M. Okutan, A. Çakir, E. Göksel, and H. Bilge, Med. Dosime. 34, 311 (2010).

[10] M. T. Tieu, P. Graham, L. Browne, and Y. S. Chin, Inter. J. Radi. Oncol. 81, 165 (2011).

[11] M. Nakano, R. F. Hill, M. Whitaker, J. H. Kim, and Z. Kuncic, J. Appl. Clin. Med. Phys. 13, 3727 (2012).

[12] K. Quach, J. Morales, M. Butson, A. B. Rosenfeld, and P. E. Metcalfe, Med. Phys. 27, 1676 (2000).

[13] I. Soong, T. Yau, C. Ho, B. Lim, S. Leung, and R. Yeung, Clin. Oncol. 16, 283 (2004).

[14] T. Vu, J. P. Pignol, E. Rakovitch, J. Spayne, and L. Paszat, Clin. Oncol. 19, 115 (2007).

[15] S. M. Bentzen, Natu. Rev. Canc. 6, 702 (2006).

[16] A. C. Shiau, M. C. Chiu, T. H. Chen, J. F. Chiou, P. W. Shueng, and S. W. Chen, Med. Dosi. 37, 417 (2013).

[17] J. Leif, H. Nguyen, A. Hollan, D. Followill, J. Galvin, and D. Kiniry, Inter. J. Radi. Onco. Biol. Phys. 90, 940 (2014).

[18] P. Scalchi, P. Francescon, and P. Rajaguru, Med. Phys. 32, 1571 (2005).

[19] I. S. Kwan, A. B. Rosenfeld, Z. Y. Qi, D. Wilkinson, M. L. Lerch, and D. L. Cutajar, Radi. Measu. 43, 929 (2008).

[20] R. Ramani, S. Russell, and P. O'Brien, Inter. J. Radi. Onco. Biol. Phys. 37, 959 (1997).

[21] Y. Akino, I. J. Das, G. K. Bartlett, H. Zhang, E. Thompson, and J. E. Zook, Med. Phys. 40, 714 (2013).

[22] S. Ito, B. C. Parker, R. Levine, M. E. Sanders, J. Fontenot, and J. Gibbons, Inter. J. Radi. Onco. Biol. Phys. 81, 584 (2011).

[23] H. F. Xiang, J. S. Song, D. W. Chin, R. A. Cormack, R. B. Tishler, and G. M. Makrigiorgos, Med. Phys. 34, 1266 (20074).

[24] J. B. Chung, J. S. Kim, I. A. Kim, and J. W. Lee, J. The Kor. Phys. Soc. 61, 1143 (2012).

[25] V. Panettieri, P. Barsoum, M. Westermark, L. Brualla, and I. Lax, Rad. Ther. Onco. 93, 94 (2009).

[26] J. C. Chow, R. Jiang, and M. K. Leung, J. Appl. Clin. Med. Phys. 12, 78 (2010).

[27] E. Healy, S. Anderson, J. Cui, L. Beckett, and A. M. Chen, J. Perks, Prac. Radi. Onco. 3, 45 (2013). 